\title{
TÉCNICAS PARA MEDICIÓN DE LA FRECUENCIA CARDÍACA
}

$\mathrm{U}$

no de los parámetros más importantes del registro electrocardiográfico consiste en la medición de la frecuencia cardíaca instantánea. Este parámetro es estimado a través de mediciones de tiempo entre cada una de las ondas R del complejo cardíaco. Por lo cual es necesario el diseño y la implementación de algoritmos para la detección de la onda R. La frecuencia cardíaca es un parámetro determinado por el tiempo entre cada latido del corazón. Este parámetro se calcula a partir del tiempo entre cada onda R del complejo cardíaco.

La medición de la frecuencia cardíaca se puede hacer a través de técnicas basadas en circuitos analógicos y digitales que captan la ocurrencia en el tiempo de una onda R del complejo cardíaco. En la actualidad predominan las estrategias fundamentadas en el tratamiento digital de señales, que utilizan técnicas basadas en filtros digitales para el realce de la onda R. Además de la señal electrocardiográfica, también es utilizada la señal de pulso oximetría. Esta señal genera una curva que está en fase con el ECG y es producto de los cambios de saturación de oxígeno en la sangre. 
La detección de la onda $\mathrm{R}$ es posible después de cumplir con la correcta implementación del sistema de acondicionamiento de señal y un conjunto de algoritmos basados en filtros digitales. Esto último, con el fin de contar con una señal ECG libre de distorsiones, ya que estas pueden impedir la correcta detección de la onda R. Además, las distorsiones pueden causar la presencia de formas de ondas similares al complejo QRS y pueden generar falsas detecciones que conlleven datos erróneos de la frecuencia cardíaca. En el caso del desplazamiento de línea de base, puede acontecer la no detección de ondas $\mathrm{R}$ existentes.

El objetivo de este capítulo es proporcionar un resumen de las diferentes técnicas para la detección de la onda $\mathrm{R}$ y la medición de la frecuencia cardíaca. En primera instancia se citarán los métodos basados en circuitos electrónicos, los cuales realizaban la detección de la onda $\mathrm{R}$ a partir de la señal ECG en versión analógica. Consecutivamente, son presentados los métodos basados en el tratamiento digital de señales, que son orientados a la manipulación de muestras obtenidas después de la conversión analógica a digital de la señal ECG.

\section{Técnicas basadas en circuitos electrónicos}

El cálculo de la frecuencia cardíaca puede ser obtenido a través de la implementación de sistemas básicos basados en circuitos electrónicos. Como se puede ver en la figura 34, de manera sucesiva a una etapa de acondicionamiento de la señal se puede conectar un sistema de umbralización y una etapa de cuantificación.

El sistema de umbralización consiste en la detección de valores de amplitud igual o por encima de un nivel de voltaje preestablecido. La etapa de cuantificación permite calcular el tiempo entre cada detección aportada por el sistema de umbralización.

El sistema de umbralización puede estar compuesto por un amplificador en configuración de seguidor de tensión que cumple la labor de generar una etapa de alta impedancia a la salida del sistema de acondicionamiento de señal [21], [22]. De manera continua se puede colocar una etapa de rectificación de media onda (figura 35). 
FIGURA 34.

SISTEMA

ELECTRÓNICO

PARA MEDICIÓN

DEL TIEMPO

ENTRE ONDAS R

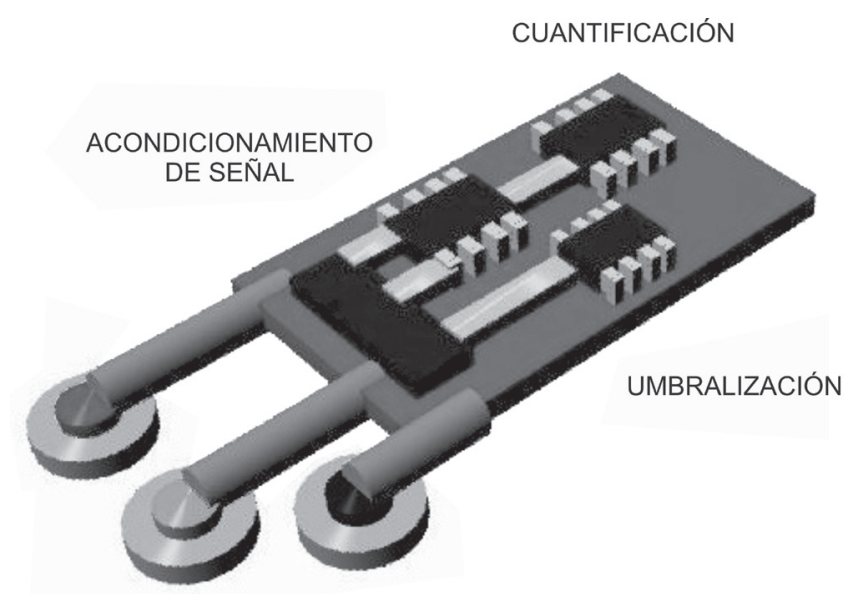

FIGURA 35.

SISTEMA DE

UMBRALIZACIÓN

FUENTE:

ELABORACIÓN

PROPIA.

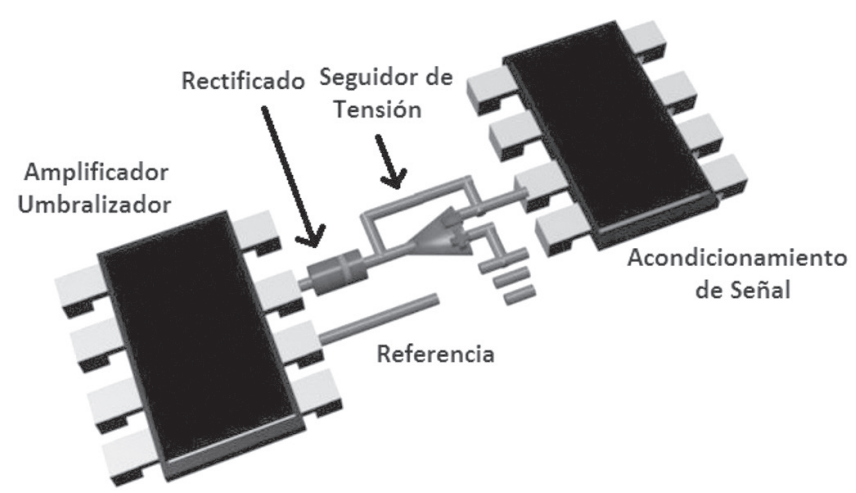

El sistema de rectificado está conectado a un amplificador umbralizador, el cual tiene el papel de generar una señal binaria en fase a la onda R del complejo cardíaco. El amplificador umbralizador posee una entrada, denominada referencia. Esta entrada tiene la función de proporcionar un valor de tensión que equivale al valor umbral.

La salida del amplificador umbralizador se compone de una señal binaria, cuyo valor unitario está en fase con la onda R del complejo cardíaco. La figura 36 ejemplifica una imagen de una señal adquirida con un osciloscopio digital, 
contiene una señal ECG y la salida de dos etapas: rectificación y umbralización.
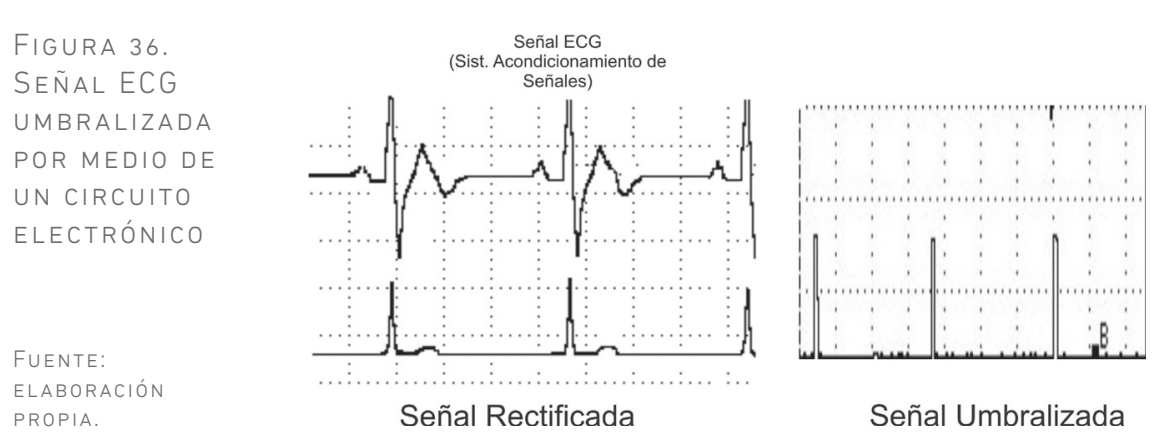

Luego de la umbralización se pasa a una etapa de cuantificación. Esta etapa permite contabilizar el tiempo entre cada valor unitario, tomando como referencia cada detección aportada por la señal umbralizada.

La etapa de cuantificación puede ser implementada mediante el uso de circuitos digitales como por ejemplo un micro controlador. La figura 37 contiene un diagrama de bloques que indica los componentes principales para la implementación de un circuito de cuantificación. Este sistema toma la señal umbralizada y la utiliza como una señal de control que proporciona el inicio y final de un contador. La salida del sistema de cuantificación es un registro que contiene el tiempo entre cada onda $\mathrm{R}$ del complejo cardíaco.

Los sistemas basados en hardware poseen algunas desventajas a la hora de realizar el proceso de umbralización de la señal ECG y la detección de la onda R. En primer lugar, no pueden adaptarse a las irregularidades de la señal ECG, ya que cada sujeto puede presentar proporciones distintas en las diferentes formas de ondas. 
FIGURA 37.

SISTEMA DE

CUANTIFICACIÓN

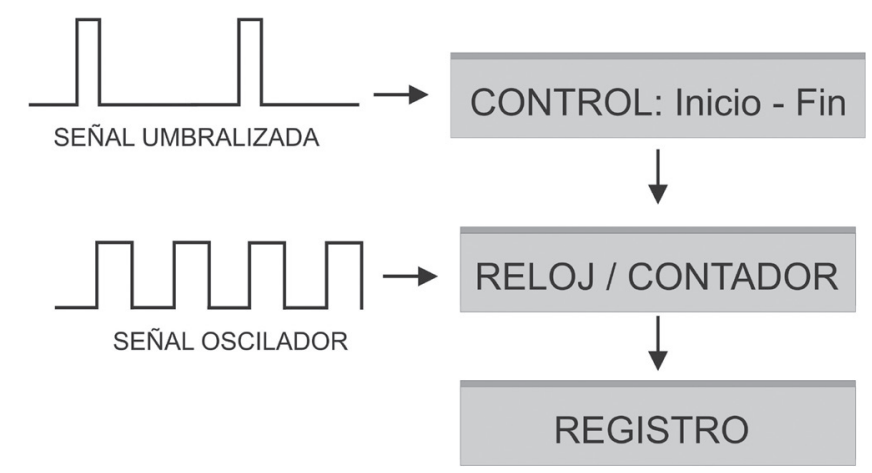

\section{Técnicas basadas en tratamiento digital de señales}

La onda R corresponde al valor positivo de mayor magnitud que se encuentra en el registro electrocardiográfico. Esta onda está precedida por la onda Q y luego aparece la onda S. La detección de la onda $\mathrm{R}$ es un proceso que permite la cuantificación de la frecuencia cardíaca instantánea y es la base de diferentes tipos de análisis del sistema cardiovascular y nervioso autónomo [36]. Al transformar la señal ECG analógica en una versión discreta, se deben conservar las propiedades de la onda $\mathrm{R}$ que la permiten diferenciarse de las demás ondas del complejo cardíaco.

En esta sección se exponen otras aplicaciones de los filtros digitales, en las cuales no se requiere eliminar o atenuar una distorsión. En este caso, los filtros digitales orientan su función a permitir la detección de una forma de onda particular.

\section{Los bancos de filtros}

En muchas ocasiones, la forma de onda particular posee componentes contenidas dentro del espectro de la señal bajo estudio. Sin embargo, no se puede tener un modelo determinístico para establecer los valores de frecuencias que limitan la ubicación en el espectro de las componentes asociadas a la forma de onda.

Los bancos de filtros son una forma eficiente de encontrar el rango de frecuencias del espectro de una señal ECG que contiene las componentes espectrales de la onda R. La estructura principal de un banco de filtros consiste 
en tener una señal de entrada $x[n]$ que es tratada por una serie de filtros con respuesta al impulso $\mathrm{H}[\mathrm{n}]$, donde $n$ indica la numeración del filtro. Esta estructura genera como salida, diferentes versiones de la señal de entrada $x[n]$. La figura 38 ilustra un diagrama de bloques de un sistema de banco de filtros.

FIGURA 38.

ESTRUCTURA DE

UN BANCO DE

FILTROS

FUENTE:

ELABORACIÓN

PROPIA.

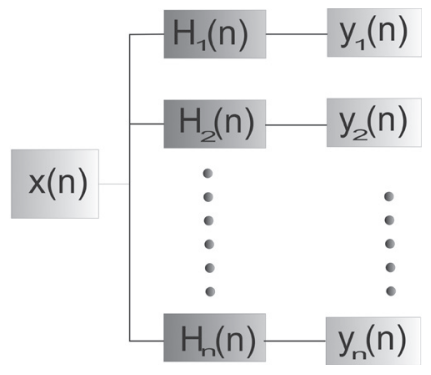

Cada filtro contenido en la estructura de este banco posee valores definidos de frecuencia de corte. Por lo general se definen como filtros pasa banda y sus respectivos valores de frecuencia de corte son consecutivos.

A través de las siguientes líneas de código en $\mathrm{Matlab}^{\circledR}$ se puede implementar un banco de filtros. El primer paso consiste en establecer el rango de frecuencias:

$$
\begin{aligned}
& F=[0.110 ; 1020 ; 2030 ; 3040] ; \\
& w n=F /(F s / 2) ;
\end{aligned}
$$

El segundo paso es generar la familia de funciones $\mathrm{H}(\mathrm{n})$ :

$$
\begin{aligned}
& \text { Fori }=1: 4 \\
& H(i,:)=\text { fir1 }\left(50, w n(i,:),{ }^{\prime}\right. \text { bandpass'); }
\end{aligned}
$$

end

Para ejecutar el banco de filtros se implementa la siguiente rutina iterativa:

$$
\text { for } n=1: 4
$$$$
X N(n,:)=\text { filter }(H(n,:),[1], x) \text {; }
$$

end

El resultado obtenido (figura 39) en el proceso está almacenado en la matriz XN. Cada fila de esta matriz contiene las diferentes versiones de la señal $x(n)$ de entrada. 
FIGURA 39.

SEÑAL ECG

PROCESADA

A TRAVÉS DE

UN BANCO DE

FILTROS

FUENTE:

ELABORACIÓN

PROPIA.
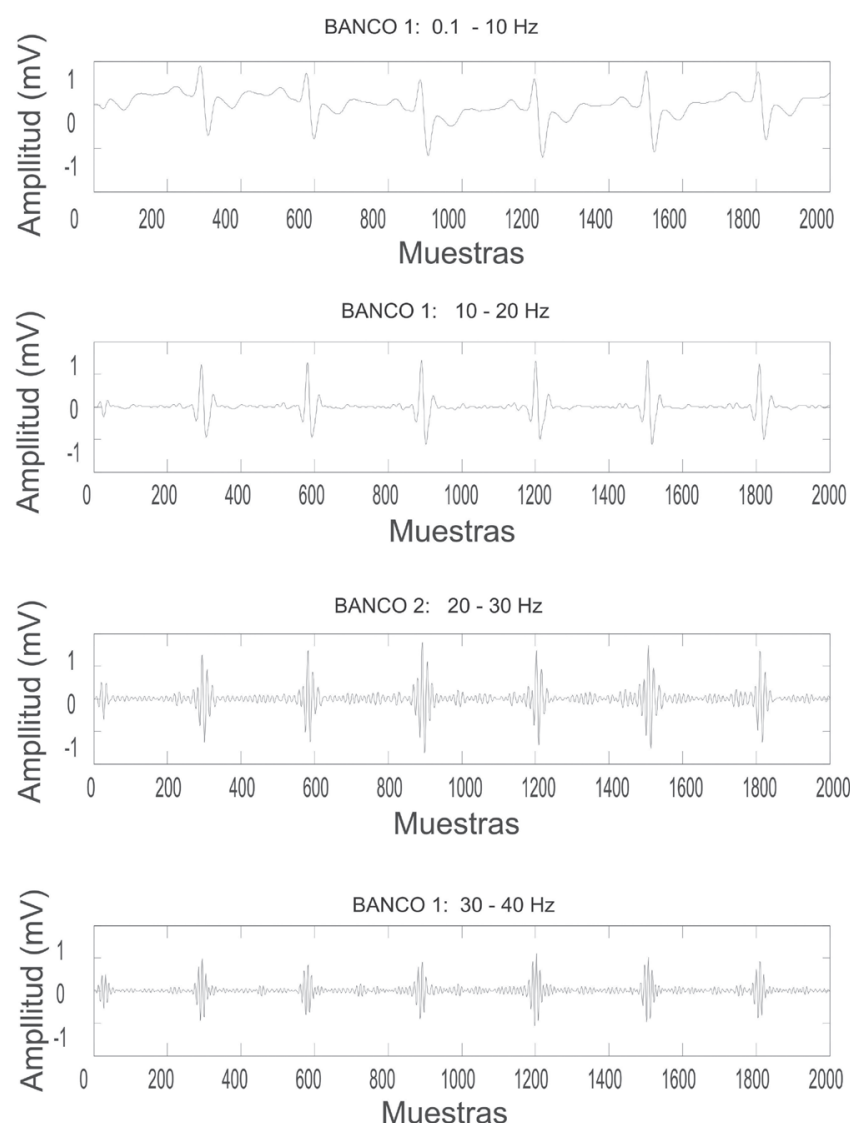

La figura 39 contiene el resultado obtenido y cada una de las versiones de la señal ECG obtenida de cada filtro del banco diseñado. Este ejercicio iterativo permite establecer una relación entre cada forma de onda visualizada en el dominio del tiempo y un conjunto de componentes espectrales delimitado por el respectivo filtro pasa banda que ha generado la versión de la señal.

En este ensayo se puede observar que la onda $\mathrm{R}$ se destaca con un incremento en amplitud en la señal obtenida en la banda entre 10 y $20 \mathrm{~Hz}$. Este resultado permite determinar el rango de frecuencias de las componentes espectrales que están asociadas a la onda $\mathrm{R}$ del complejo cardíaco.

La ventaja de los sistemas para detección de la onda $\mathrm{R}$, basados en tratamiento digital de señales, radica en su gran potencial para discriminar 
componentes espectrales. Esta capacidad permite detectar la onda R en presencia de ruido y las respectivas distorsiones que pueden ser causadas a la señal ECG.

\section{La transformada Wavelet continua}

El método basado en bancos de filtros permite una asociación entre formas de onda y espectro de la señal. Cómo método alternativo para lograr esta asociación están las transformadas tiempo-frecuencia, que están orientadas a permitir de manera simultánea la visualización de los planos del tiempo y de la frecuencia en análisis de señales. Como un caso de éxito se expone a continuación la transformada Wavelet continua.

La transformada Wavelet continua posee ventajas con respecto a la transformada de Fourier. La principal ventaja consiste en tener un grupo de funciones que son de gran parecido a la onda $\mathrm{R}$ y facilitan su correlación. Para los sistemas de detección de la onda $\mathrm{R}$, basados en algoritmos de tratamiento digital de señales, adquieren mayor robustez con la transformada Wavelet al permitir la detección de diferentes variaciones de la onda $\mathrm{R}$.

La transformada Wavelet continua está basada en el estudio de señales por medio de un proceso de descomposición. En este proceso, la señal bajo estudio es comparada con una función base denominada Wavelet [37]. Esta estrategia ha sido utilizada para la detección de formas de ondas particulares en contextos como la señal electrocardiográfica y las señales de potencia eléctrica.

Una de las funciones wavelets más conocidas es la función sombrero mexicano. Esta forma de onda se describe en la ecuación 5.

ECUACIÓN 5.

$$
\varphi(t)=\left(1-t^{2}\right) e^{\frac{t^{2}}{2}}
$$

La figura 40 ilustra la representación en el dominio del espacio de la función sombrero mexicano. 
FIGURA 40.

FUNCIÓN

SOMBRERO

MEXICANO

FUENTE:

ELABORACIÓN PROPIA.

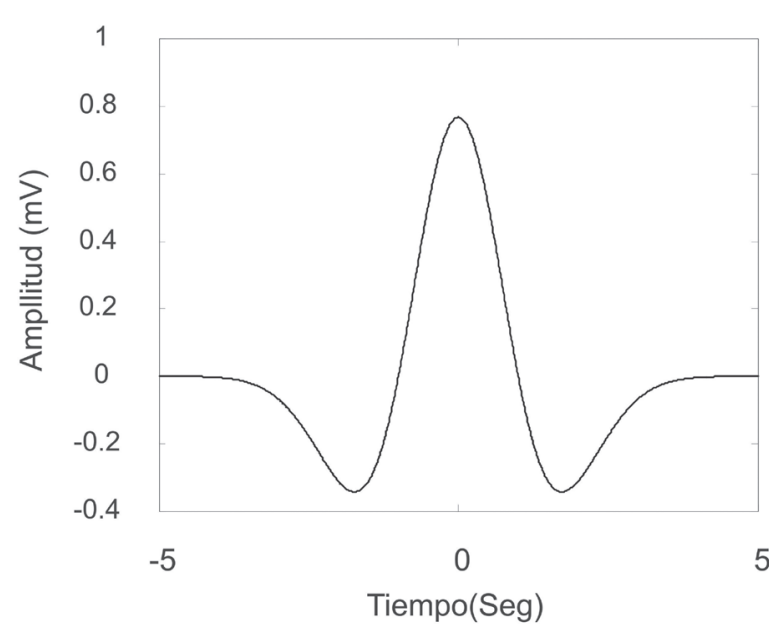

5

Dentro del procedimiento de ejecución de la transformada Wavelet, es importante tomar la función base y generar una familia con variaciones de escala de tiempo. Las escalas de tiempo permiten tener variaciones de duración de la función base y da la oportunidad de tener una mayor cantidad de señales de referencia para realizar comparaciones exhaustivas de la señal bajo estudio. Al haber escogido la función wavelet madre, se procede a generar una familia de funciones que resultan de desplazamientos y escalas en el dominio de la frecuencia (ecuación 6).

ECUACIÓN 6.

$$
\varphi_{u, s=\frac{1}{\sqrt{s}} \varphi}\left(\frac{t-u}{s}\right)
$$

Se puede generar en Matlab ${ }^{\circledast}$ una simulación de esta función en el dominio del tiempo para diferentes escalas de frecuencia de la función sombrero mexicano. En estas líneas se declara el arreglo de datos que determina las escalas de la función base:

$$
\begin{aligned}
& T s=0.01 ; \\
& F s=1 / T s ;
\end{aligned}
$$


$t=-10: T s: 10$;

$L=$ length $(t)$;

$f=0.01: 0.05: 0.2$;

$N=$ length $(f)$;

En las siguientes líneas se ejecutan las iteraciones que generan cada versión escalada de la función sombrero mexicano.

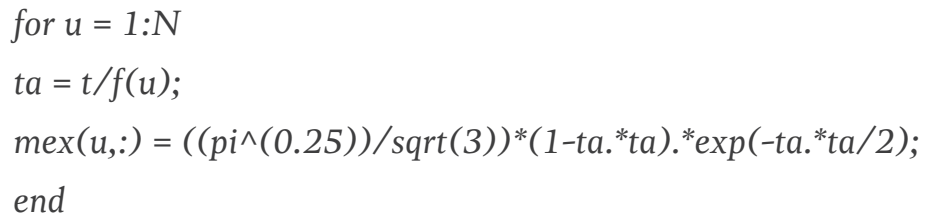

figure

$\operatorname{mesh}(t, f, \operatorname{mex})$

La figura 41 contiene el resultado del proceso de escalamiento de la función sombrero mexicano.

\section{FIGURA 41.}

FUNCIÓN

SOMBRERO

MEXICANO

ESCALADA

FUENTE:

ELABORACIÓN PROPIA.

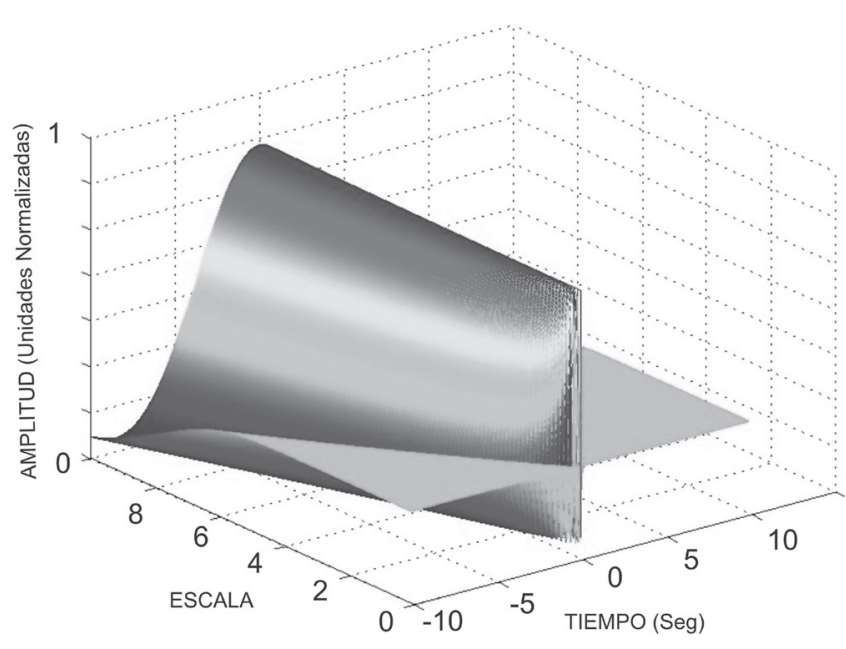

Para ejecutar el análisis basado en la transformada Wavelet se recurre a la ecuación 7. Esta expresión permite que cada una de las versiones escaladas de la función Wavelet se opere sobre la función de entrada. La respuesta de esta operación proporciona una función bidimensional con dos ejes: tiempo y frecuencia (escala). 
ECUACIÓN 7 .

$$
W F(u, s)=\int_{-\infty}^{+\infty} x(t) \frac{1}{\sqrt{s}} \varphi\left(\frac{t-u}{s}\right) d t
$$

A través del siguiente código en Matlab $^{\circledR}$, se implementa el proceso de comparación utilizando el comando filter:

$$
\begin{aligned}
& \text { for } u=1: N \\
& Q=\text { filter }(\operatorname{mex}(u,:), 1, x) ; \\
& T W(u,:)=Q(1000: 1998) ;
\end{aligned}
$$

End

figure

$\operatorname{mesh}(t(1000: 1998), f, T W)$

El resultado obtenido está contenido en la figura 42. Se puede apreciar que los picos de amplitud aparecen en fase con las ondas $\mathrm{R}$ de una señal electrocardiográfica. Esto es producto de la correlación entre la función Wavelet sombrero mexicano y el complejo QRS de la señal electrocardiográfica.

\section{FIGURA 42. \\ TRANSFORMADA WAVELET DE UNA SEÑA ELECTROCAR- DIOGRÁFICA \\ FUENTE: \\ ELABORACIÓN PROPIA.}

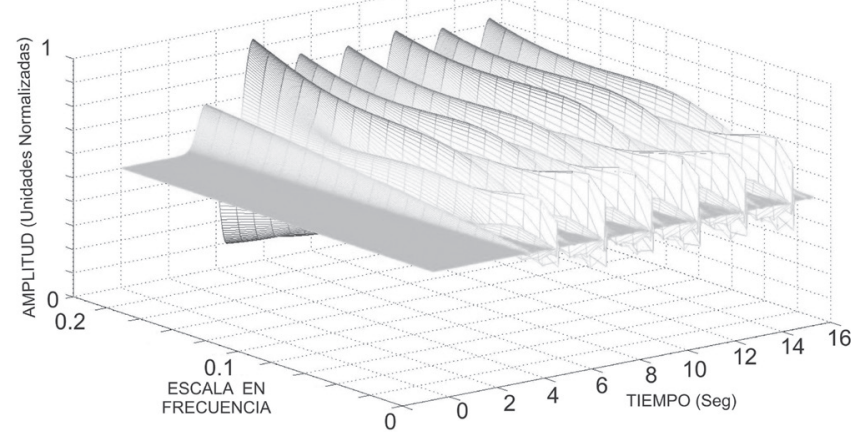

La interpretación del resultado visualizado en la figura 39 es de carácter fundamental para el entendimiento de la transformada Wavelet. En el eje escala en frecuencia se pueden apreciar los resultados de la correlación obtenida entre la señal de entrada y cada versión de la función base escogida. 
Entre mayor sean las amplitudes de los picos obtenidos, mayor será la probabilidad de obtener la detección de un patrón. El patrón detectado será parecido a la forma de la función wavelet escogida. El eje del tiempo sirve para establecer el instante en el cual existe el parecido entre la escala de la función base y la señal bajo estudio.

Por tal razón, uno de los aportes de la transformada Wavelet es permitir al usuario observar simultáneamente el eje de tiempo y escala en frecuencia.

\section{El proceso de umbralización}

Después del proceso de detección o realce de la onda $\mathrm{R}$ del complejo cardíaco, se implementan una serie de pasos propuestos por el autor Tompkins W. [17], [18]. En primera instancia, Tompkins sugiere utilizar un filtro pasa banda con frecuencias de cortes centradas en el valor de frecuencia $12 \mathrm{~Hz}$. Este filtro pasa banda se establece con el objetivo de obtener las componentes asociadas a la onda R. La figura 43 ilustra el resultado obtenido al utilizar una señal ECG tomada del repositorio Physionet.
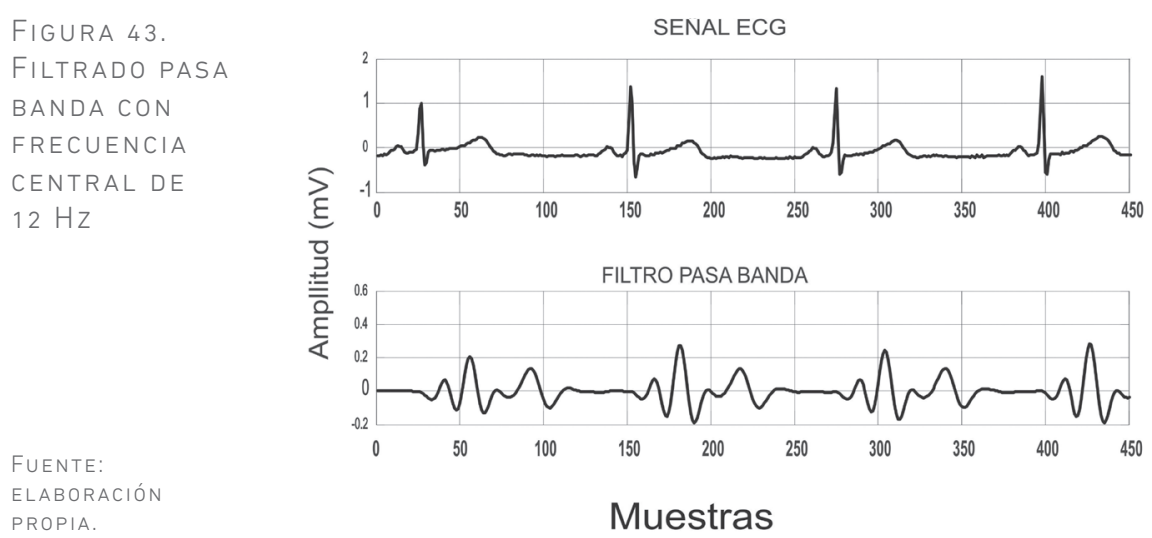

El siguiente paso propuesto consiste en aplicar una serie de filtros derivadores basados en la primera y segunda derivada de la señal ECG obtenida del filtrado pasa banda. El procedimiento tiene como entrada la variable ec que contiene la señal electrocardiográfica. Como salidas se obtienen dos señales: 
D1 y D2, que corresponden a la primera y segunda derivada de la variable ec. Este procedimiento se implementa a través de las siguientes líneas de código:

$$
\begin{aligned}
& B 0=\left[\begin{array}{lll}
1 & 0 & -1
\end{array}\right] ; \\
& \text { D1 = filter (B0,[1],ec); } \\
& D 1=a b s(D 1) \text {; } \\
& B 1=\left[\begin{array}{lll}
1 & -2 & 0
\end{array}\right] \text {; } \\
& \text { D2 = filter (B1,[1],ec); } \\
& \text { D2 = abs (D2); }
\end{aligned}
$$

La figura 44 permite ver en el dominio del tiempo la magnitud de la primera y segunda derivada de la señal electrocardiográfica.

FIGURA 44.

PRIMERA Y SEGUNDA DERIVADA DE UNA SEÑAL ECG

FUENTE:

ELABORACIÓN PROPIA.
PRIMERA DERIVADA

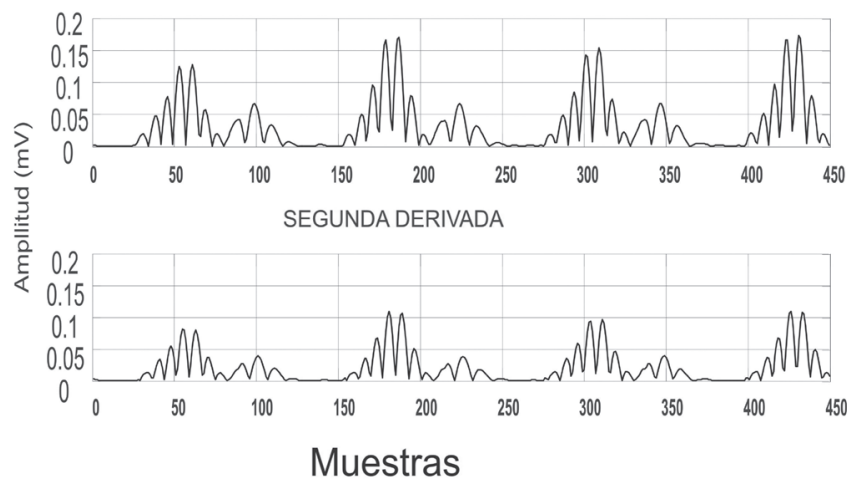

Al tomar la señal que equivale a la primera derivada (D1) y la equivalente a la segunda derivada (D2) se procede a realizar el cálculo de la nueva señal contenida en la variable Y2. En las siguientes líneas de código se calcula la variable con la nueva señal:

$$
y 2=1.3^{*} \mathrm{D} 1+1.1^{*} \mathrm{D} 2 \text {; }
$$

El resultado obtenido al sumar las operaciones de diferenciación se puede observar en la figura 45.

Por último se procede a un proceso de suavizado utilizando un filtro promediador o integrador. El código para implementar el proceso de suavizado es el siguiente: 
$L=16$;

$B p=(1 / L) *$ ones $(1, L)$

$y p=$ filter $(B p, 1, y)$;

El proceso de suavizado genera la variable yp que corresponde a una versión de la señal ECG, en la cual el pico de mayor amplitud indica la presencia de una onda R (figura 46).

FIGURA 45.

SUMA DE LA

PRIMERA Y

SEGUNDA

DERIVADA DE UN ECG

FUENTE:

ELABORACIÓN

PROPIA.

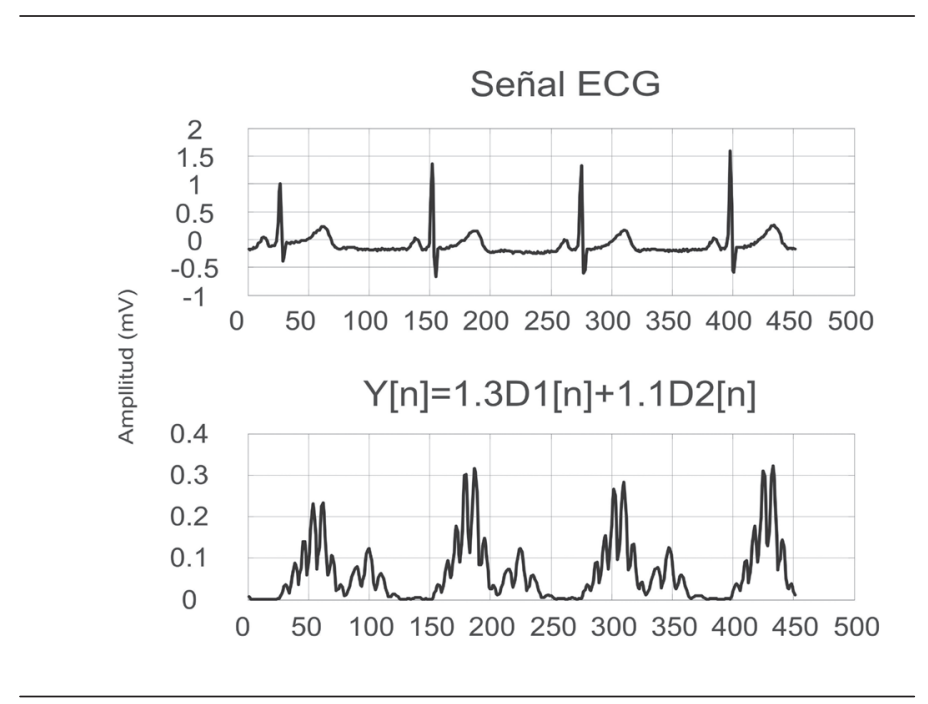

FIGURA 46.

PROCESO DE

PROMEDIADO

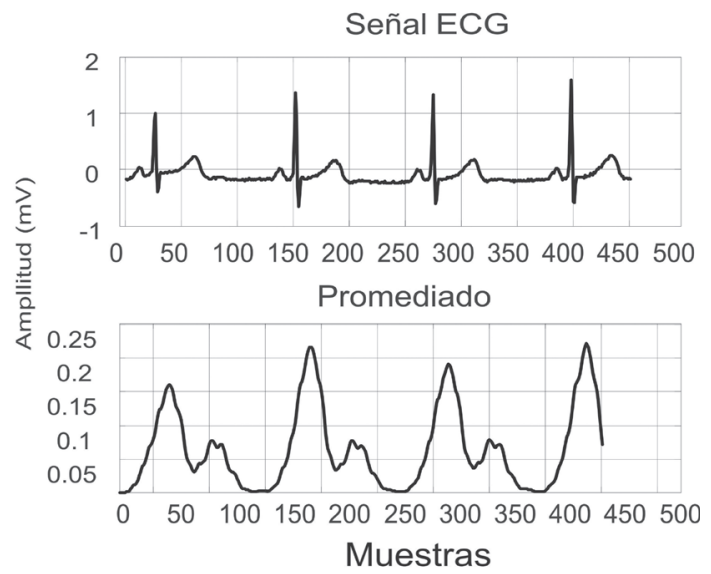


Para poder cuantificar el tiempo entre cada onda R se procedió a la aplicación de un umbral a la señal promediada. En el siguiente código se realiza una operación de normalización a la variable yp, con la finalidad de obtener una señal con solo valores entre 0 y 1 . Posteriormente se aplica un operador de umbral:

$$
\begin{aligned}
& M=\max (y p) ; \\
& y p=y p / M ; \\
& y u=y p>0.6 ;
\end{aligned}
$$

El resultado obtenido en el proceso de umbralización está contenido en la figura 47.

FIGURA 47.

UMBRALIZACIÓN

DE LA SEÑAL

ECG
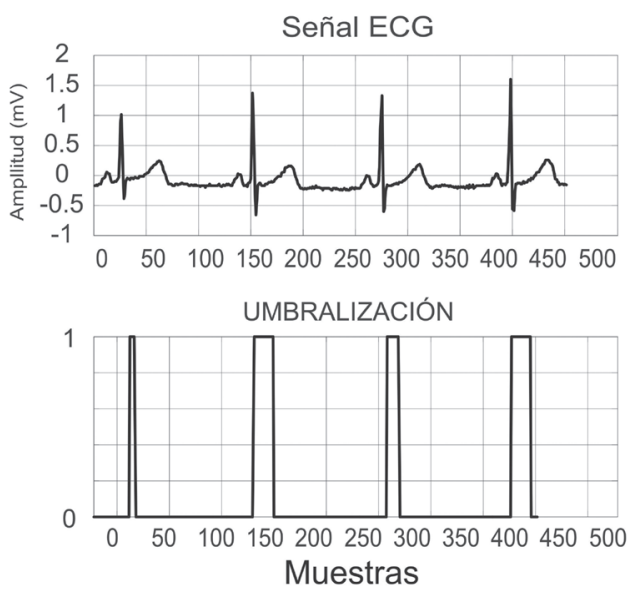

\section{Umbralización adaptativa}

Dentro del proceso de umbralización de la señal ECG es de gran utilidad el cálculo del histograma. Esta herramienta estadística permite obtener un análisis de la frecuencia de ocurrencia de los diversos valores de amplitud. En la figura 48 se puede ver un ejemplo de una señal ECG después del filtrado pasa banda centrado en la frecuencia de $12 \mathrm{~Hz}$ y su respectivo histograma. 
FIGURA 48. HISTOGRAMA DE LA SEÑAL ECG

FUENTE: ELABORACIÓN PROPIA.

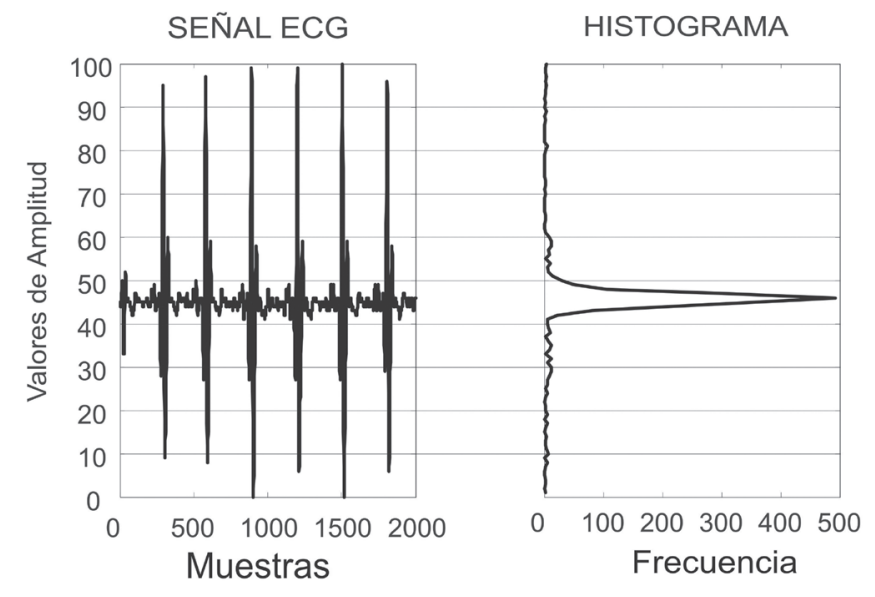

El histograma posee dos ejes, el eje de referencia representa todos los valores de amplitud que se pueden presentar en la señal bajo estudio. En el caso de la figura 45 ha sido visualizado de tal manera que el eje de referencia se graficó de manera vertical.

El otro eje del histograma es el de la frecuencia y contiene la cantidad de veces que aparece cada valor de amplitud en la señal bajo estudio. En la figura 48 se puede ver este eje de manera horizontal.

Trabajos previamente publicados han aprovechado la información contenida en el histograma para establecer el valor umbral de la señal ECG [26]. En estos trabajos se usa el cálculo de la segunda derivada del histograma, tal como se ilustra en la figura 49 .

El histograma y su segunda derivada (figura 46) pueden ser relacionados con diferentes aspectos de la señal ECG. Como es el caso del nivel de offset, el cual puede ser identificado a través del pico de mayor amplitud en el histograma de la señal ECG. Los valores de amplitud de las ondas P Y R aparecen con un valor de amplitud de frecuencia menor a los asociados con el nivel de offset.

En el cálculo de la segunda derivada, el primer cero que aparece después del pico positivo de mayor amplitud puede ser tomado como referencia para encontrar el valor umbral. 
FIGURA 49.

CÁLCULO DE

LA SEGUNDA

DERIVADA DEL

HISTOGRAMA DE

LA SEÑAL ECG
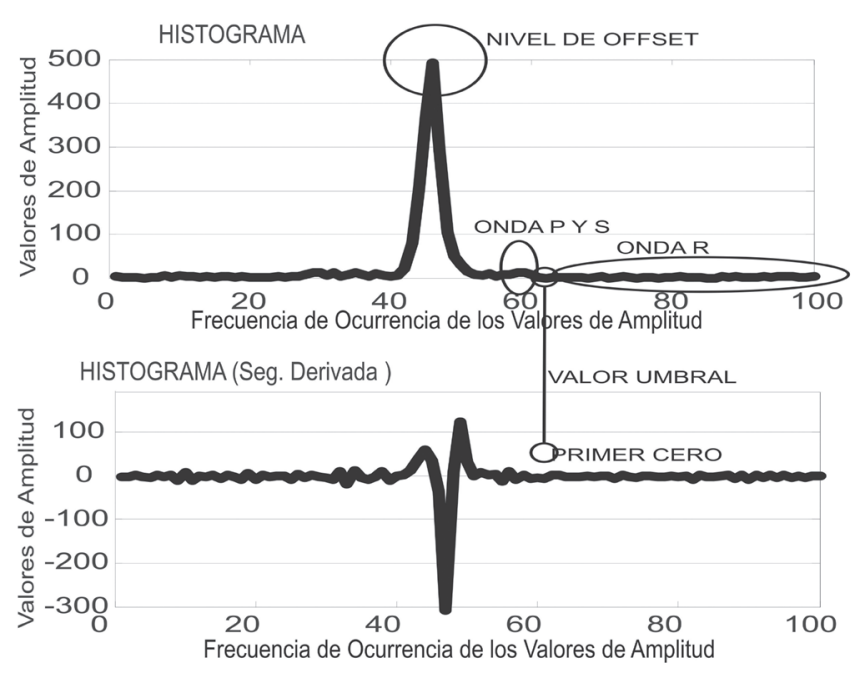

Está técnica conlleva una estrategia adaptativa para el cálculo del valor umbral, debido a que el histograma varía de acuerdo con los cambios de valores de amplitud de la señal ECG. Por lo cual ofrece una gran ventaja con respectos a los métodos clásicos de umbralización, los cuales no están diseñados para contemplar el comportamiento no determinístico que contienen la señale ECG.

A continuación se expone una función en Matlab, denominada ECGHISTO. Esta función es propuesta para tomar como entrada una señal ECG almacenada en la variable $b$ y la respectiva frecuencia de muestreo Fs. Los parámetros de salida corresponden a la señal umbralizada que contiene el valor de 1 en cada ocurrencia de la onda R y 0 para los demás valores. En la salida de la función ECGHISTO se encuentra el valor umbral y el histograma de la señal (Variable $h$ ).

Function $[a 1, u m b r a l, h]=$ ECGHISTO $(b, F s)$

$\%$ en esta parte del código se tiene un filtro pasa banda y permite aislar componentes de frecuencia ajenas a la onda $\mathrm{R}$.

$$
\begin{aligned}
& f l=12 ; \\
& f h=14 ; \\
& w 1=f l /(F s / 2) ;
\end{aligned}
$$




$$
\begin{aligned}
& w 2=f h /(F s / 2) ; \\
& B=\operatorname{fir} 1(30,[w 1 w 2]) ; \\
& a=\operatorname{filter}(B, 1, b) ;
\end{aligned}
$$

\% De manera consecutiva se procede a normalizar la señal ECG filtrada y se convierte en un arreglo de datos con valores enteros.

$$
\begin{aligned}
& m 1=\max (a) ; \\
& a=a / m 1 ; \\
& a=a^{*} 100 ; \\
& C=\operatorname{length}(a) ; \\
& a=\operatorname{round}(a) ; \\
& m=\min (a) ; \\
& a=a+a b s(m) ; \\
& a=a+1 ; \\
& F=\max (a) ;
\end{aligned}
$$

\% se genera una matriz llena de ceros con la intención de poder cuantificar la ocurrencia de cada valor de amplitud.

$$
\begin{aligned}
& A=\operatorname{zeros}(F, C) ; \\
& \text { for } i=1: C \\
& q=a(i) \\
& A(q, i)=1 ;
\end{aligned}
$$

End

\% En esta sección del código se calcula el valor consolidado del histograma.

$$
\begin{aligned}
& \text { for } i=1: F \\
& h(i)=\operatorname{sum}(A(i,:)) ; \\
& \text { end }
\end{aligned}
$$

\% La última parte de este código calcula la segunda derivada del histograma y ubica la posición, en el arreglo de datos, el valor umbral.

$$
h \max =\max (h) \text {; }
$$

$h p o s=f i n d(h==h \max )$;

$K=1: F$;

$h d=\operatorname{diff}(h, 2)$;

$h d=\left[\begin{array}{lll}0 & 0 & h d\end{array}\right]$;

$Z=$ find $(h d==1)$; 
$z$ pos $=$ find $(z>h p o s)$;

umbral $=z(z p o s(1))$;

$a 1=a>$ umbral;

\section{La señal de ritmo cardíaco}

La señal de ritmo cardíaco es una serie de tiempo que contiene la información de los intervalos entre cada onda $\mathrm{R}$ del complejo cardíaco. Las unidades de este tipo de señal están en segundos y corresponden al tiempo transcurrido entre cada una de las ondas. El inverso matemático de estos valores corresponde al término frecuencia cardíaca instantánea.

La señal de ritmo cardíaco se puede extraer a partir del proceso de umbralización (figura 35). Como se pudo observar, se cuenta con un tren de impulsos de valor unitario.

En primera instancia se toma la variable yu que contiene la señal umbralizada y se procede con la instrucción find para establecer la ubicación de cada muestra de la señal que posee un valor igual a uno.

$\%$ posiciones de los 1

$y 1=$ find $(y u==1)$;

Como se pudo observar en la anterior línea de código, la variable y1 corresponde a un arreglo de datos que contiene las posiciones con valor igual a uno que debe tener la variable $y 1$.

Posteriormente se procede a estimar las posiciones iniciales de los trenes de impulsos. Este procedimiento se realiza con el fin de establecer el inicio de una onda R. Las siguientes líneas de código toman la variable y1 para originar los arreglos $\mathrm{y}_{1 \mathrm{a}} \mathrm{Y}_{\mathrm{lb}}$, para generar la variable $y 2$ :

$$
\begin{aligned}
& y 1 a=\left[y 1^{\prime} 0\right] ; \\
& y 1 b=\left[\begin{array}{ll}
0 & y
\end{array}{ }^{\prime}\right] ; \\
& y 2=y 1 a-y 1 b ;
\end{aligned}
$$

La variable y2 permite establecer la distancia en muestras entre cada posición que contiene un valor unitario. Cuando existen dos posiciones consecutivas, la variable y2 tomará el valor de uno. En el caso que existan dos posiciones lejanas, la variable y 2 tendrá un valor mayor de uno. Este escenario se debe interpretar como el hallazgo de un nuevo tren de impulsos, el 
cual indica el inicio de una nueva onda R. El siguiente paso es extraer estas posiciones de inicio de tren de impulsos, lo cual se puede realizar a través de la siguiente instrucción:

$P=$ find $(y 2>1)$;

Posteriormente se procede a calcular la cantidad de muestras entre cada inicio de los trenes de impulsos. Mediante las siguientes líneas de código se genera la variable pos, que contiene las posiciones que indican el inicio de cada tren de impulsos:

$$
\begin{aligned}
& L 1=\text { length }(p) ; \\
& \text { fori=1:L1 } \\
& C=p(i) ; \\
& \text { pos }(i)=y 1(C) ; \\
& \text { end } \\
& \text { pos1=[pos 0]; } \\
& \text { pos2=[ } 0 \text { pos }] ; \\
& \operatorname{pos} 3=\operatorname{pos} 1-\operatorname{pos} 2 ; \\
& \operatorname{pos} 4=\operatorname{pos} 3(1: L 1) ;
\end{aligned}
$$

La variable pos 4 contiene la cantidad de muestras entre cada inicio de tren de impulsos. Esto es equivalente a la distancia entre cada onda R del complejo cardíaco. Para obtener el valor de la distancia en unidades de tiempo, se debe multiplicar cada valor de la variable pos 4 por el periodo de muestreo Ts:

$$
\begin{aligned}
& T s=0.008 ; \\
& \text { tiempo=pos } 4^{*} T s ;
\end{aligned}
$$

La variable tiempo expresa, en unidades de segundos, la distancia entre cada onda R. Este arreglo de datos contiene los valores denominados: Tiempos R-R. Estos valores son el componente de la señal de ritmo cardíaco y la base de estudio para el análisis de la variabilidad de la frecuencia cardíaca.

En este capítulo se ha expuesto el concepto de frecuencia cardíaca, el cual consiste en una medición del tiempo entre la ocurrencia de cada onda R del complejo cardíaco. En primera instancia, es de gran importancia establecer un método para la detección de la onda R, para lo cual se han resumido los aportes, en cuanto a técnicas basadas en circuitos electrónicos y filtrado digital. La detección de la onda $\mathrm{R}$ es un proceso que requiere de la correcta obtención de 
la señal ECG libre de distorsiones, debido a la presencia de formas de ondas no deseadas que pueden causar la errónea detección de patrones.

El uso de un filtrado pasa banda es un método sugerido en los diferentes estilos de técnicas para la detección de la onda R. La contribución de este tipo de filtros consiste en aislar componentes espectrales de otras formas de ondas. Como una contribución de este texto, se resalta el uso del histograma del ECG que permite conocer la distribución estadística de los valores de amplitud. 
